\title{
Differences between Fallers by Tripping and Nonfallers in the OSFS Test
}

\author{
Sohee Shin ${ }^{*}$, Shinichi Demura ${ }^{2}$, Tsuneo Watanabe1, Bateer Shi1, Tamotsu Yabumoto1, \\ Toshio Matsuoka ${ }^{1}$ \\ ${ }^{1}$ Department of Sports Medicine and Sports Science, Gifu University Graduate School of Medicine, Gifu, Japan \\ ${ }^{2}$ Kanazawa University Graduate School of Natural Science \& Technology, Kanazawa, Japan \\ Email: sohee@gifu-u.ac.jp
}

Received 2 April 2014; revised 3 May 2014; accepted 13 May 2014

Copyright (C) 2014 by authors and Scientific Research Publishing Inc.

This work is licensed under the Creative Commons Attribution International License (CC BY). http://creativecommons.org/licenses/by/4.0/

(c) (i) Open Access

\section{Abstract}

This study evaluates the effects of a fall experience caused by tripping during the repetitive stepping movements over an obstacle [obstacle-single leg forward step (OSFS) test]. The study included 147 participants who were divided into 2 groups: 25 fallers caused by tripping and 122 non- fallers. The subjects were asked to step forward over a 10-cm-high obstacle with 1 leg and then return to their original position, as quickly as possible, and this test was repeated for 5 times. The OSFS test was evaluated in 2 phases: the OSFS-F phase, wherein the participants stepped forward on one leg, and the OSFS-R phase, wherein they returned to their original position. Significant differences were observed in both phases of the OSFS test between the two groups, and the fallers by tripping were significantly inferior to the nonfallers. The area under the curve [AUC; area under the receiver operating characteristic (ROC) curve] was more than 0.63 for all the parameters, which was statistically significant. In conclusion, the fallers by tripping were inferior to the nonfallers in the obstacle step movement.

\section{Keywords}

Fallers by Tripping, Nonfallers, ROC Curve, OSFS Test

\section{Introduction}

According to the reports by Suzuki et al. [1] and Demura et al. [2], which researched the conditions related to falling, the fall incidence rate for one year was approximately $20 \%$ in the community-dwelling elderly aged 65 and over, although regional differences were observed. Because fall incidence increases with age, this increases

*Corresponding author. 
the possibility of the elderly sustaining fractures and becoming bedridden. A decline in physical fitness seriously limits the activities of daily living (ADL) for the elderly and increases the possibility of a fall. Therefore, it is important for the elderly to adequately evaluate their physical fitness levels in order to achieve an independent life without falls.

Suzuki et al. [3] evaluated the life function and physical functions of the community-dwelling elderly using the Tokyo Metropolitan Institute of Gerontology Index and reported that the scores of fallers were higher than those of nonfallers. In addition, Tinetti et al. [4] reported that the elderly with high activity levels have a lower fall incidence. Therefore, a decline in the ability to achieve ADL may be closely related to fall risk.

The mechanisms that can lead to falls in the elderly include tripping, slipping, misstepping, and staggering. Nevertheless, several differences exist between the respective fall mechanisms and optimal prevention strategies. According to previous studies [5] [6] that reviewed these causes, falling was mostly caused by tripping during walking. Tripping easily occurs through difficulty of foot lifting caused by decreased leg strength, decreased vision, and recognition of obstacles.

On the other hand, falls caused by "sliding," "staggering," "dizziness," and "swaying” primarily occur because of environmental factors and medical history. Hence, an individual risk profile is necessary to effectively prevent falling [2]. Most falls occur when individuals with internal factors, including decreased physical function and decreased performance of physical activities, are affected by external factors such as an obstacle; therefore, it is important to observe each individual in detail to understand which factors increase the risk of falling on an individual basis.

To date, several fall-related physical fitness tests have been developed to predict fall risk. To approach fall prevention from various view points, it is necessary to understand which body functions are evaluated in these field tests. For example, because the elderly, who were judged to extensively sway during standing on the basis of the COP (center of pressure) measurements, are unable to maintain a stable posture as well, they may exhibit high possibility of falling. In addition, the elderly with inferior leg strength are unable to support their body when stepping forward quickly and thus may fall down.

Shin and Demura [7]-[11] developed the obstacle-single leg forward step (OSFS) test which evaluates the fall-related physical fitness of the elderly. This test comprises the movements whereby participants step forward over an obstacle with the one leg and return the leg to its original position [7]-[9]. The present study conducted the OSFS test to investigate nonfallers and fallers due to tripping.

This study aimed to examine the efficacy of the OSFS test in evaluating the performance characteristics of the elderly who are likely to trip using the receiver operating characteristic (ROC) analysis.

\section{Methods}

\subsection{Participants}

In total, 147 healthy elderly females who could independently walk participated in this study; among these, 25 participants (77.2 \pm 5.0 years) were fallers who fell by tripping in the past year and the other 122 participants (75.3 \pm 6.0 years) were nonfallers. Prior to the measurements, the purpose and procedures of this study were explained to all the participants in detail, and informed consent was obtained from them. This study was approved by the institutional review board of the Gifu University School of Medicine (reference number, 24-310).

\subsection{Obstacle-Single Leg Forward Step Test (0-SFS Test; Figure 1)}

The participants stood barefoot with relaxed arms on a step sheet in a quiet room. They were asked to view the obstacle. Before the measurement was recorded, we determined the leg that was easier to stand on and operate for each participant according to the assessment method by Demura et al. [12]. The participants stood on the supporting leg, stepped forward over the obstacle with the other leg, and then returned to the original position as quickly as possible; this exercise was repeated 5 times. The step width from the start spot was $25 \mathrm{~cm}$, and the obstacle (height, $10 \mathrm{~cm}$ ) was set at the midway point. The measurements were recorded in a single trial following a practice trial, and the mean value was used for statistical analysis. A tester controlled the obstacle lightly so that it did not move from the set place or fall down if the participants tripped over it. The step test was performed using the step sheet T.K.K.5301 (Takei inc. Shinsyu, Japan), which uses foot pressure to measure the swing time in 1 leg from rising to landing. The parameters measured were the time of a forwarding phase 


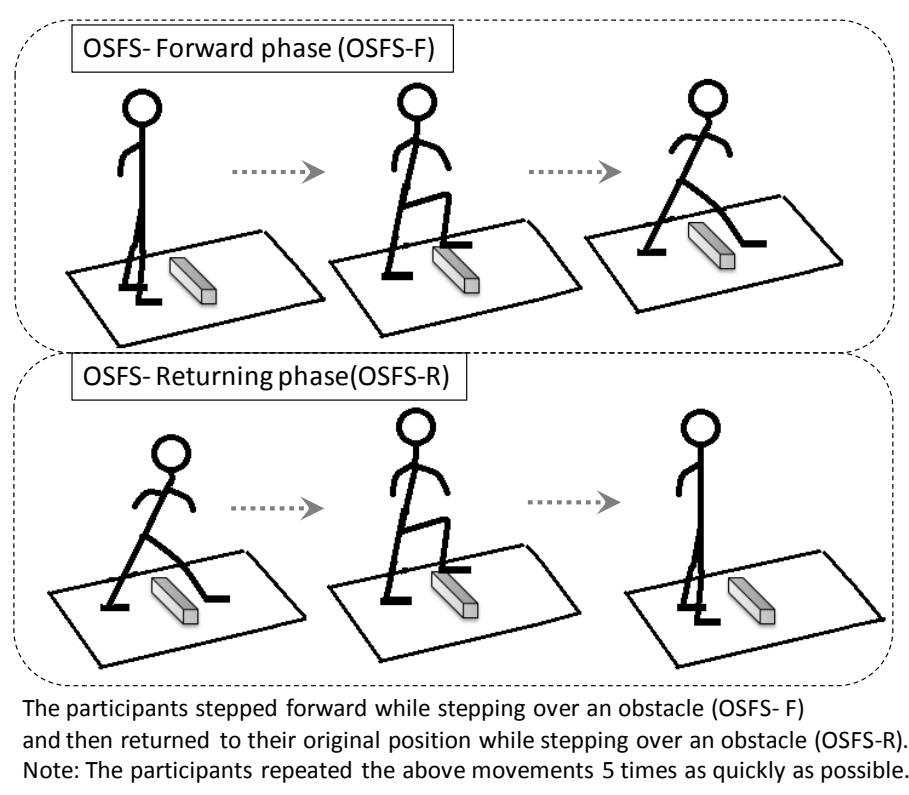

Figure 1. Obstacle single leg forward step test (OSFS test).

(OSFS-F: the mean time of stepping forward) and a returning phase (OSFS-R: the mean time of returning to the original position), and a mean time of both phases.

\subsection{Statistical Analysis}

To assess the mean differences between the fallers by tripping and the nonfallers for the OSFS test along with the physical characteristics, unpaired t-tests were used. To evaluate the efficacy of the OSFS test, an ROC curve was used. The probability level of $p<0.05$ was indicative of statistical significance.

\section{Results}

Table 1 presents the mean differences in the physical characteristics and the scores of activity of daily living (ADL) questionnaire from the Ministry of Education, Culture, Sports, Science and Technology Japan. Age, height, weight, and ADL scores revealed no significant differences between the groups. However, the effect sizes revealed relatively lower values $(0.17-0.35)$.

Table 2 presents the mean differences between the groups for the OSFS test parameters. Significant differences were observed in all the parameters, and the fallers by tripping were significantly inferior to the nonfallers. Further, the effect sizes exhibited moderately high values $(0.62-0.86)$.

Table 3 presents the results of AUC calculation of the ROC curve in the OSFS test. The AUCs of the ROC curves in the OSFS parameters were statistically significant $(0.63-0.71)$.

\section{Discussion}

To evaluate the elderly with lower physical fitness, very safe tests should be selected. In addition, it is preferable that the test content relates closely to their ADL and is available for rehabilitation and functional recovery [13]. This study conducted an OSFS test for nonfallers and fallers by tripping and evaluated the efficacy of this test to assess the performance characteristics of the elderly who trip easily.

Causes of falls in the elderly are classified into variable and nonvariable factors. It is important to understand in advance whether it is possible to improve the fall-related characteristics of the elderly in order to prevent falls [14]. The present study focuses on the variable factors of fall risk, particularly movement characteristics of the elderly who trip easily.

This study investigated the fall experiences of elderly participants in the past year and included the movements that led to the fall. It was determined that 25 of 50 fallers fell down by tripping. Although this number 
Table 1. Differences between the fallers by tripping and the nonfallers in each parameter.

\begin{tabular}{|c|c|c|c|c|c|c|}
\hline \multirow[b]{2}{*}{ Parameters } & \multicolumn{3}{|c|}{ Descriptive statistics } & \multicolumn{2}{|c|}{ Unpaired t-test } & \multirow[b]{2}{*}{ ES } \\
\hline & Group & Mean & SD & t-value & p-value & \\
\hline \multirow[t]{2}{*}{ Age } & Faller by tripping & 77.2 & 5.0 & 1.50 & 0.13 & 0.31 \\
\hline & Nonfaller & 75.3 & 5.9 & & & \\
\hline \multirow[t]{2}{*}{$\mathrm{ADL}$} & Faller by tripping & 23.8 & 5.2 & 1.72 & 0.09 & 0.35 \\
\hline & Nonfaller & 25.5 & 4.5 & & & \\
\hline \multirow[t]{2}{*}{ Height } & Faller by tripping & 148.4 & 5.3 & 0.82 & 0.41 & 0.17 \\
\hline & Nonfaller & 147.3 & 6.0 & & & \\
\hline \multirow[t]{2}{*}{ Weight } & Faller by tripping & 52.1 & 9.3 & 1.54 & 0.13 & 0.32 \\
\hline & Nonfaller & 49.3 & 7.9 & & & \\
\hline
\end{tabular}

ES (Effect size), faller by tripping 25 persons, nonfaller 122 persons.

Table 2. Differences between the fallers by tripping and the nonfallers in the OSFS test.

\begin{tabular}{cccccccc}
\hline & \multicolumn{5}{c}{ Descriptive statistics } & \multicolumn{2}{c}{ Unpaired t-test } \\
\hline Parameters & Group & Mean & SD & t-value & p-value & ES \\
\hline OSFS (Mean) & Faller by tripping & 0.67 & 0.18 & 2.79 & 0.01 & 0.84 & $*$ \\
& Nonfaller & 0.56 & 0.10 & & & 0.62 & $*$ \\
OSFS & Faller by tripping & 0.63 & 0.19 & 2.14 & 0.04 & & \\
Forward phase & Nonfaller & 0.55 & 0.11 & & & \\
OSFS & Faller by tripping & 0.69 & 0.20 & 2.97 & 0.01 & $*$ \\
Backward phase & Nonfaller & 0.57 & 0.11 & & & & $*$ \\
\hline
\end{tabular}

* $\mathrm{p}<0.05$, ES (Effect size), faller by tripping 25 persons, nonfaller 122 persons.

Table 3. The area under an ROC curve (AUC).

\begin{tabular}{ccccc}
\hline Parameters & AUC & SE & P-value & $95 \%$ CI \\
\hline OSFS Mean & 0.69 & 0.06 & $0.00^{*}$ & $0.58-0.80$ \\
OSFS Forward phase & 0.63 & 0.06 & $0.05^{*}$ & $0.50-0.75$ \\
OSFS Backward phase & 0.71 & 0.05 & $0.00^{*}$ & $0.61-0.81$ \\
\hline
\end{tabular}

${ }^{*} \mathrm{P}<0.05$ faller by tripping 25 persons, nonfaller 122 persons.

was relatively lower than that reported in a previous study [15], it infers that many falls are attributed to tripping. Falls by tripping have a higher prevention possibility via improvement of ADL or visual function compared with falls caused by other reasons such as "slipping," "staggering," "dizziness," and "swaying." Weerdesteyn et al. [16] evaluated the different approaches toward the trip avoidance process and reported that the success rate of stepping over an obstacle significantly increased by performing a unique program for 5 weeks, which included performing multiple tasks at once, and obstacle courses, which simulate ADL. In addition, Lamoureux et al. [17] reported that the obstacle avoidance ability of the elderly significantly improved by performing resistance exercises such as flexion and extension movements of hip, knee, and ankle joints for 24 weeks.

The OSFS test used in the present study consisted of very simple movements whereby participants step forward over an obstacle with 1 leg and then return the leg to its original position. However, this test indicated that the fallers by tripping took longer to perform the OSFS movements compared with the nonfallers. In addition, the ROC analysis results indicated that AUC was significantly large, and it was found that the OSFS test is useful in distinguishing between fallers by tripping and nonfallers.

The OSFS test was constructed after considering the following 4 points: 1) when almost falling, the elderly quickly step forward 1 step to maintain their base of support (BOS) and to prevent the fall; 2) a decrease in leg strength and balance ability as well as the range of motion (ROM) of the hip, knee, and ankle joints are asso- 
ciated with fall occurrence; 3) the elderly frequently fall by tripping over an obstacle or a step; and 4) the screening test used should consist of safe movements that the elderly can easily understand. The OSFS test requires the elderly to step over the obstacle while supporting the body with 1 leg and to return it to the original position. Hence, this test may be difficult to perform for the elderly with decreased physical fitness when compared with other tests comprising activities such as walking and one-leg standing.

The fallers by tripping were found to be inferior to the nonfallers in both phases of the OSFS test (OSFS-F: the phase of stepping forward and OSFS-R: the phase of returning to the original position). Further, the effect size in the OSFS-R phase was relatively larger compared with that of the OSFS-F phase (ES: OSFS-B $=0.86$, OSFS-F = 0.62). Shin et al., [18] reported that there were no significant differences between the fallers and nonfallers in the OSFS-F phase. Therefore, a fall risk may be better explained by the OSFS-R phase values, where the stepped leg is returned to the original position compared with the OSFS-F phase values, where the subject is stepping over an obstacle. The elderly with decreased leg strength are generally unable to stretch their legs when their body is inclined forward because of the fear of falling forward [19]. This indicates that a lack of the ability to brace one's legs may cause falls.

Meanwhile, the OSFS-F phase also indicated a significant difference in this study. The fallers by tripping may take a long time to step over an obstacle regardless of the phases (OSFS-F or OSFS-R). Watanabe [20] reported that the elderly are more likely to disrupt body balance in conditions that require posture change such as stepping over obstacles. Particularly, it is inferred that the elderly who trip easily used a higher clearance than necessary for the obstacle because they cannot smoothly elevate the leg by flexion movements of the hip, knee, and ankle joints. In addition, such elderly people may find it difficult to maintain physical balance when standing on 1 leg while stepping over the obstacle. Therefore, the elderly may require a longer time when shifting to the next movement after stepping forward or after returning to the original position.

\section{Conclusion}

The fallers by tripping require a longer time in both the OSFS-F and OSFS-B phases. The OSFS test may be useful to discriminate between the fallers by tripping and the nonfallers because a large AUC was found.

\section{References}

[1] Suzuki, T. (2000) Questionnaire for Falls Assessment of Elderly People and Its Application. Health Assessment Manual Kosei Kagaku Kenkyusho, 142-163.

[2] Demura, S., Sato, S., Shin, S. and Uchiyama, M. (2012) Setting the Criterion for Fall Risk Screening for Healthy Community-Dwelling Elderly. Archives of Gerontology and Geriatrics, 54, 370-373. http://dx.doi.org/10.1016/j.archger.2011.04.010

[3] Suzuki, T. (2003) Epidemiology and Implications of Falling among the Elderly. Nippon Ronen Igakkai Zasshi, 40, 85-94. http://dx.doi.org/10.3143/geriatrics.40.85

[4] Tinetti, M.E. (1986) Performance Oriented Assessment of Mobility Problems in Elderly Patients. Journal of the American Geriatrics Society, 34, 119-126.

[5] Suzuki, T. (2001) Fall Accidents of the Elderly. Journal of Clinical Rehabilitation, 10, 955-960.

[6] Yasumura, S. and Shinno, N. (1997) Risk Factors for Falls among Elderly People. Journal of Physical Therapy, 14, 199-205.

[7] Shin, S. and Demura, S. (2007) Effective Tempo of the Step Test for Dynamic Balance Ability in the Elderly. Journal of Physiological Anthropology, 26, 563-567. http://dx.doi.org/10.2114/jpa2.26.563

[8] Shin, S. and Demura, S. (2009) The Relationship of Age and Leg Strength in the Step Test with Stipulated Tempo in the Elderly. Achives of Gerontology and Geriatrics, 49, 311-316. http://dx.doi.org/10.1016/j.archger.2008.11.009

[9] Shin, S. and Demura, S. (2009) Relationship between the Step Test with Stipulated Tempos and Gait Ability in the Elderly. Journal of Physiological Anthropology, 28, 49-54. http://dx.doi.org/10.2114/jpa2.28.49

[10] Shin, S. and Demura, S. (2010) The Usefulness of Single Leg Forward Step Test for the Evaluation of the Elderly's ADL Ability. Japanese Journal of Test of Evaluation of Health, Physics, Education, 10, 27-32.

[11] Shin, S. and Demura, S. (2010) Comparison and Age-Level Differences among Various Step Tests for Evaluating Balance Ability in the Elderly. Achives of Gerontology and Geriatrics, 50, 51-54. http://dx.doi.org/10.1016/j.archger.2009.05.007

[12] Demura, S., Sato, S. and Sugiura, H. (2010) Lower Limb Laterality Characteristics Based on the Relationship between 
Activities and Individual Laterality. Gazzeta Medica Italiana, 169, 181-191.

[13] Demura, S., Shin, S. and Yamaji, S. (2008) Sex and Age Differences of Relationships among Stepping Parameters for Evaluating Dynamic Balance in the Elderly. Journal of Physiological Anthropology, 27, 207-215. http://dx.doi.org/10.2114/jpa2.27.207

[14] Demura, S. (2013) Fall Prevention for Healthy Community-Dwelling Elderly. Kyorin Press, Tokyo.

[15] Akiyama, T., Fukushima, T., Hisasita, H. and Ebina, H. (1996) Study on Own-Injuries of the Elderly in Outdoor Walk Space. The 16th Report of Japan Society of Traffic Engineers, 193-196.

[16] Weerdesteyn, V., Rijken, H., Geurts, A.C., Smits-Engelsman, B.C., Mulder, T. and Duysens, J. (2006) A Five-Week Exercise Program Can Reduce Falls and Improve Obstacle Avoidance in the Elderly. Gerontology, 52, 131-141. http://dx.doi.org/10.1159/000091822

[17] Lamoureux, E., Sparrow, W.A., Murphy, A. and Newton, R.U. (2003) The Effects of Improved Strength on Obstacle Negotiation in Community-Living Older Adults. Gait and Posture, 17, $273-283$. http://dx.doi.org/10.1016/S0966-6362(02)00101-7

[18] Shin, S. and Demura, S. (2011) Examination of the Usefulness of the Obstacle-Single Leg Forward Step (OSFS) Test for Evaluating Fall Risk. Advances in Physical Education, 1, 7-10. http://dx.doi.org/10.4236/ape.2011.12002

[19] Mano, Y. (2008) Falls in the Elderly and Measurements. Ishiyaku Press, Tokyo.

[20] Watanabe, K. (1993) A Response to an Obstacle in When the Elderly Walk. Health Care, 35, 93-97. 